\title{
Coefficients of Restitution Based on a Fractal Surface Model
}

Equations of rigid-body mechanics provide a means to predict the post-collision behavior without recourse to highly complex, detailed analysis of deformations during contact. Before the prediction can be completed, the coefficient of restitution, which relates the rebound velocity to the incident velocity, must be estimated properly. The coefficient of restitution depends on the surface topography in addition to the material properties and incident velocity. Recent investigations showed that surface topography can be characterized properly by fractal models. This paper proposes a normal contact model for a fractal surface in contact with a rigid smooth half-space. The fractal surface is constructed based on the Cantor set and composed of elastic-perfectly plastic material. Asymptotic continuous expressions for the load-displacement relations during loading and unloading are derived. Based on these results, we study the effects of surface roughness, material properties and incident velocity on the coefficient of restitution.

[DOI: $10.1115 / 1.1574063$ ]

\section{Introduction}

The analysis of impact phenomena has important applications in different fields. For example, impacts between the slider and disk greatly influence the reliability of magnetic disk drives. Accurate analysis of the collision between two deformable bodies is difficult and time-consuming even for the large-scale computational capabilities developed recently. If the local deformations at the contact area and the transient response during collision are not of interest, the colliding bodies can be treated as rigid for predicting the post-collision behavior. In this case, a coefficient of restitution, purports to describe the energy loss during collision, must be incorporated to relate the rebound velocity to the incident velocity. When the impact is perpendicular to the nominal plane of the interface, the coefficient of restitution $e$ is defined to be the ratio of the rebound velocity to the incident velocity. Values of $e=1$ and $e=0$ denote the idealized concepts of perfectly elastic and plastic impacts, respectively. Introduction of the coefficient of restitution greatly simplifies the procedure for determining the post-collision motion. The success of the rigid-body impact analysis depends on the correct estimation of the coefficient of restitution.

An important subject is how the coefficient of restitution is related to the basic physical material properties. Tabor [1], Goldsmith [2], and Johnson [3] considered the impact between a sphere and a stationary half-space at moderate impact velocities. Both colliding bodies were assumed to be smooth. As indicated by Johnson [3], when the impact velocity is small compared with the elastic wave velocity, the static contact force-compression law can be employed to investigate the impact behavior. Under this condition, the coefficient of restitution can be estimated based on principles of energetics. Up to the instant of maximum compression, the initial kinetic energy transforms into strain energy, elastic and plastic, of the two colliding bodies. After the point of maximum compression, there is a release of elastic stresses and the kinetic energy of rebound is equal to the work done during elastic recovery. If the compliance relationship of load and displacement

\footnotetext{
${ }^{1}$ To whom correspondence should be addressed.

Contributed by the Applied Mechanics Division of THE AMERICAN SOCIETY OF MECHANICAL ENGINEERS for publication in the ASME JOURNAL OF APPLIED MECHANICS. Manuscript received by the ASME Applied Mechanics Division, October 10, 2001; final revision, September 9, 2002. Associate Editor: K. T. Ramesh. Discussion on the paper should be addressed to the Editor, Prof. Robert M. McMeeking, Department of Mechanical and Environmental Engineering University of CaliforniaSanta Barbara, Santa Barbara, CA 93106-5070, and will be accepted until four months after final publication of the paper itself in the ASME JOURNAL OF APPLIED MECHANICS.
}

for elastic-plastic contact is known, the rebound velocity and hence the coefficient of restitution can be determined. However, the compliance relationship for elastic-plastic contact is not precisely defined, so that a theory of elastic-plastic impact is necessarily approximate. By choosing suitable compliance relationships, the dependence of the coefficient of restitution on the material properties, incident velocity, and radius of the ball can be estimated, [1-3].

All engineering surfaces are rough in nature and have random height distribution, [4,5]. The compliance relationship of real bodies depends closely on the waviness and roughness of the contact surfaces, [3]. Therefore, it stands to reason that surface topography of contact bodies will have a large effect on the collision process, especially at the initial stage of compression. Several probabilistic theories have been developed to model the mechanical behavior of contacting rough surfaces, [6-11]. One of the most popular models is the Greenwood and Williamson (G\&W) model, [6]. In this model, the rough surface is represented by a collection of hemispherical asperities having a constant radius of curvature. The heights of the summits are distributed normally about the mean asperity plane and it is assumed that the contacting asperities deform elastically according to Hertz theory. Chang et al. [12] modified the G\&W model and proposed an elasticplastic contact model of rough surfaces. Based on this model, Chang and Ling [13] derived the relationship between the coefficient of restitution and the surface topography. However, recent studies showed that conventional statistical parameters used to characterize the surface topography, including the summit radius in the G\&W model, are not unique to a surface. These parameters depend on the resolution and scan length of the roughnessmeasuring instrument, [14-17]. This result suggests the use of fractal geometry, [18], for the characterization of surface roughness. Lately several fractal surface models have been proposed to describe the interaction between rough surfaces in different areas, [17,19-24]. Majumdar and Bhushan [21] used the WeierstrassMandelbrot function to simulate surface roughness and employed modified Hertz equations to model the elastic-plastic deformation of the surface. Borodich and Mosolov [23] constructed a fractal rigid die using the Cantor set. They derived asymptotic compliance expressions for the fractal die penetrating a rigid-perfectly plastic half-space and elastic half-space, respectively. Warren et al. [24] generalized the method of Borodich and Mosolov [23] by allowing the fractal surface to deform. A continuous asymptotic model incorporating volume conservation was developed to describe the rigid-perfectly elastic deformation of a fractal surface in contact with a smooth and rigid half-space. Although 
much work has been done on the normal contact behavior between rough surfaces using fractal surface model, little attention has been devoted to the significance of the fractal parameters on the coefficient of restitution.

The purpose of this paper is to investigate the effect of surface roughness on the coefficient of restitution. We consider a fractal surface composed of elastic-perfectly plastic material in contact with a smooth rigid half-space. Following Borodich and Mosolov [23] and Warren et al. [24], the fractal surface is constructed based on the Cantor set. Continuous asymptotic compliance relations during loading and unloading are derived, respectively. These relations are then used to express the coefficient of restitution in terms of surface topography and material properties, in addition to impact velocity.

\section{Cantor Set Surface Model}

The Cantor set surface shown in Fig. 1 is constructed by joining the segments obtained from successive stages of the Cantor set. At each stage, the middle sections of the previous segments are removed such that the total length of the remaining segments is $1 / f_{x}$ times that of the previous segments. The recess depth at the $(n$ $+1)$ th stage is $1 / f_{z}$ times that at the $n$th stage. Therefore, the horizontal length at the $n$th stage is

$$
l_{n}=\left(1 / f_{x}\right) l_{n-1}=\left(1 / f_{x}\right)^{n} l_{0},
$$

while the recess depth is

$$
h_{n}=\left(1 / f_{z}\right) h_{n-1}=\left(1 / f_{z}\right)^{n} h_{0} .
$$

As shown by Borodich and Mosolov [23], the fractal dimension $D$ can be related to the fractal parameters $f_{x}$ and $f_{z}$ as

$$
D=1-\frac{\ln f_{z}}{\ln \left(2 f_{x}\right)}+\frac{\ln 2}{\ln \left(2 f_{x}\right)},
$$

where $1<f_{x}$ and $1<f_{z}<2$. The fractal dimension $D$ along with the parameters $l_{0}, h_{0}, f_{x}$, and $f_{z}$ can be determined experimentally from a surface profile of the rough surface using the method proposed by Warren et al. [24].

\section{Normal Contact Model}

Consider a Cantor set fractal surface of unit depth in contact with a smooth rigid half-space as shown in Fig. 2. The rough surface is modeled as a Winkler foundation [25] of thickness $H^{*}$ and composed of elastic-perfectly plastic material with Young's modulus $E$, yield stress $\sigma_{y}$, and yield strain $\varepsilon_{y}=\sigma_{y} / E$. A load is applied to press the rigid half-space into the rough surface and then removed gradually. The loading and unloading process can be considered as quasi-static. Assume that each asperity behaves

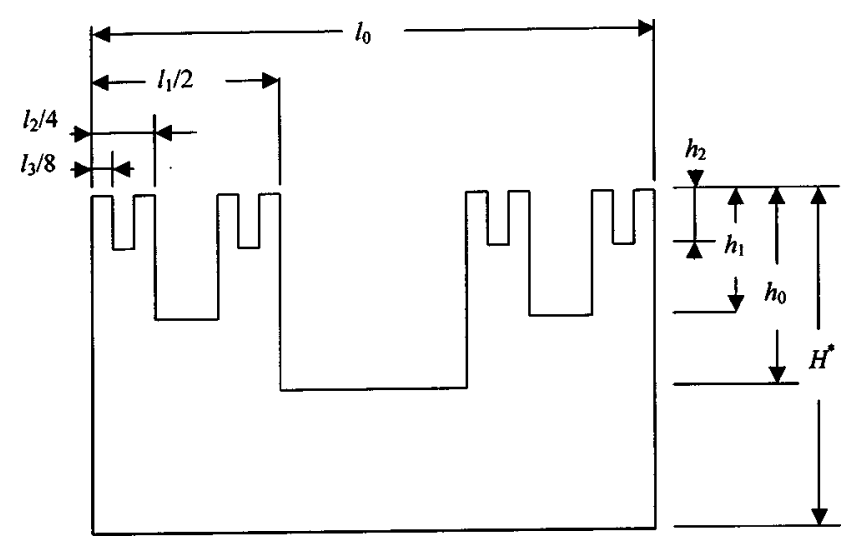

Fig. 1 Fractal surface constructed from the Cantor set

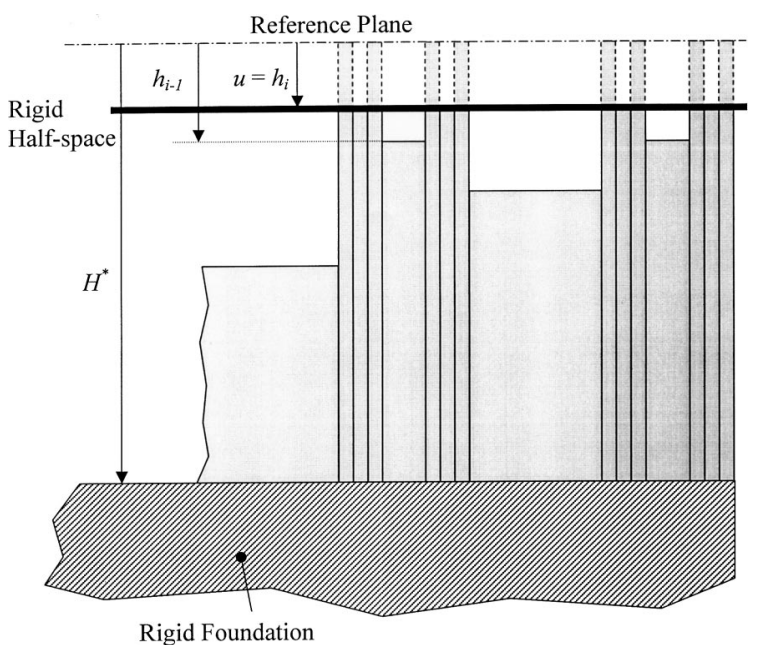

Fig. 2 A Cantor set surface in contact with a smooth rigid half-space

as an axially loaded rod and there is no interaction between the rods. Thus the total load applied to the rigid half-space is the sum of the contact load of each asperity.

3.1 Load-Unload Behavior of a Single Rod. Consider the load-unload behavior of a single asperity first. The displacement of the rigid half-space is measured from a reference plane that is $H^{*}$ apart from the bottom of the rod (Fig. 3). The distance between the top of the rod and the reference plane is $h_{i}$. When the rigid half-space with a displacement $u$ is in contact with the rod, the strain of the rod is

$$
\varepsilon_{i}=\frac{u-h_{i}}{H^{*}-h_{i}} .
$$

In the elastic region, $\varepsilon_{i}<\varepsilon_{y}$, the contact load $P_{i}$ is

$$
P_{i}=E \varepsilon_{i} s,
$$

where $s$ denotes the contact area. It can be easily shown that the inception of plastic deformation occurs when the displacement of the rigid plane reaches $u_{y}$,

$$
u_{y}=\varepsilon_{y}\left(H^{*}-h_{i}\right)+h_{i} .
$$

For $u>u_{y}$, the deformation is uniformly plastic and the corresponding contact load is

$$
P_{i}=\sigma_{y} s=E \varepsilon_{y} s .
$$

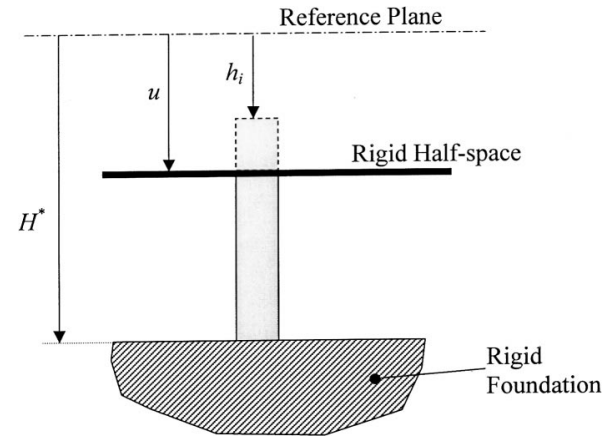

Fig. 3 A single asperity in contact with a smooth rigid halfspace 
Then consider the unloading behavior of the rod. Assume that the rod is unloaded from a maximum strain $\varepsilon_{\max }>\varepsilon_{y}$. Let $\sigma_{u}$ and $P_{u}$ indicate the stress and load during the unloading process, respectively. Then

$$
P_{u}=\sigma_{u} s .
$$

Due to the plastic deformation, there is a residual strain $\varepsilon_{r}$ when the load is removed. In the unloading stage, $\sigma_{u}$ is related to $\varepsilon$ by

$$
\sigma_{u}=E\left(\varepsilon-\varepsilon_{r}\right) .
$$

Using the fact that $\sigma_{u}=\sigma_{y}$ when $\varepsilon=\varepsilon_{\max }$, it follows that

$$
\varepsilon_{r}=\varepsilon_{\max }-\varepsilon_{y} .
$$

Thus, the dependence of the contact load during unloading on the displacement has the form

$$
P_{u}=\sigma_{y} s\left[\frac{u-u_{\max }}{\left(H^{*}-h_{i}\right) \varepsilon_{y}}+1\right] .
$$

3.2 Load-Unload Behavior of the Fractal Surface. First, consider the loading process. When the rigid half-space reaches the $n$th stage of asperities, the asperities above the $n$th stage are compressed. The strain of the $i$ th stage of asperities can be obtained by replacing $u$ in Eq. (4) with $h_{n}$ as

$$
\varepsilon_{i}=\frac{h_{n}-h_{i}}{H^{*}-h_{i}} .
$$

It can be shown that $\varepsilon_{i}>\varepsilon_{j}$, for $i>j>n$. Therefore, when some stages of asperities deform plastically, we can find a critical stage, the $n_{c}$ th stage, such that the asperities above the critical stage deform plastically while those below the critical stage deform elastically. Then the contact load $P_{n}$ can be expressed as

$$
P_{n}=\sum_{i=n+1}^{n_{c}} E \varepsilon_{i} s_{i}+\sum_{i=n_{c}+1}^{\infty} \sigma_{y} s_{i},
$$

where $s_{i}$ denotes the contact area of the $i$ th stage of asperities. The first term on the right-hand side of Eq. (13) represents the contact force due to elastic deformation, while the second term due to plastic deformation. By using Eq. (12), the elastic contact force can be expressed as

$$
\sum_{i=n+1}^{n_{c}} E \varepsilon_{i} s_{i}=\sum_{i=n+1}^{i=n_{c}} E s_{i} \frac{h_{n}-h_{i}}{H^{*}-h_{n}}=\frac{E}{H^{*}} \sum_{i=n+1}^{i=n_{c}} s_{i} \times \frac{h_{n}-h_{i}}{\left(1-\frac{h_{i}}{H^{*}}\right)} .
$$

Assuming that $h_{i} \ll H^{*}$ and neglecting the higher-order terms, the above equation reduces to

$$
\sum_{i=n+1}^{n_{c}} E \varepsilon_{i} s_{i} \approx \frac{E}{H^{*}} \sum_{i=n+1}^{n_{c}} s_{i}\left(h_{n}-h_{i}\right)
$$

Substituting Eq. (14) in Eq. (13) yields the contact force as

$$
P_{n}=\frac{E}{H^{*}} \sum_{i=n+1}^{n_{c}} s_{i}\left(h_{n}-h_{i}\right)+\sigma_{y} \sum_{i=n_{c}+1}^{\infty} s_{i} .
$$

This equation contains two different forms of series as $\sum s_{i}$ and $\sum h_{i} s_{i}$. From the definition of Cantor set and using Eqs. (1) and (2), these two series can be expressed as

$$
\begin{aligned}
& \sum_{i=n_{1}}^{n_{2}} s_{i}=\sum_{i=n_{1}}^{n_{2}}\left(l_{i}-l_{i+1}\right)=l_{n_{1}}-l_{n_{2}+1} \\
& \sum_{i=n_{1}}^{n_{2}} h_{i} s_{i}=h_{0} l_{0}\left(1-\frac{1}{f_{x}}\right)\left[\frac{\beta^{n_{1}}-\beta^{n_{2}+1}}{1-\beta}\right]
\end{aligned}
$$

where

$$
\beta=1 /\left(f_{x} f_{z}\right) .
$$

By using these relations and choosing suitable upper and lower limits for each series, Eq. (15) can be rewritten as

$$
\begin{aligned}
\frac{P_{n}}{P_{Y}}= & \frac{h_{0}}{\varepsilon_{y} H^{*}}\left\{\left(\frac{1}{f_{z}}\right)^{n}\left[\left(\frac{1}{f_{x}}\right)^{n+1}-\left(\frac{1}{f_{x}}\right)^{n_{c}+1}\right]\right. \\
& \left.-\left(1-\frac{1}{f_{x}}\right)\left(\frac{\beta^{n+1}-\beta^{n_{c}+1}}{1-\beta}\right)\right\}+\left(\frac{1}{f_{x}}\right)^{n_{c}+1},
\end{aligned}
$$

where

$$
P_{Y}=\sigma_{y} l_{0} .
$$

The total contact force for purely elastic deformation can be obtained by letting $n_{c} \rightarrow \infty$ in Eq. (19) and the result is

$$
\frac{P_{n}}{P_{Y}}=\frac{h_{0}}{\varepsilon_{y} H^{*}}\left[\left(\frac{1}{f_{z}}\right)^{n}\left(\frac{1}{f_{x}}\right)^{n+1}-\left(1-\frac{1}{f_{x}}\right)\left(\frac{\beta^{n+1}}{1-\beta}\right)\right] .
$$

Then, we proceed to study the unloading behavior of the fractal surface. Let $u_{\max }$ denote the maximum displacement of the rigid half-space, and $\bar{n}_{c}$ the corresponding critical stage. That is to say, when $u=u_{\max }$, asperities above the $\bar{n}_{c}$ th stage have plastic contact while those below the $\bar{n}_{c}$ th stage have elastic contact. For the elastically deformed asperities, the load-displacement curve in the unloading process coincides with that in the loading process. On the other hand, the load-displacement relationship of the plastically deformed asperities during unloading is described by Eq. (11). When the rigid half-space is withdrawn to a stage below the critical stage, say the $n$th stage, the total contact load can be expressed as

$$
P_{u}=\sum_{i=n+1}^{\bar{n}_{c}} E s_{i} \frac{u-h_{i}}{H^{*}-h_{i}}+\sum_{i=\bar{n}_{c}+1}^{\infty} E s_{i}\left(\frac{u-u_{\max }}{H^{*}-h_{i}}+\varepsilon_{y}\right) .
$$

For stages above the critical stage, due to the plastic deformation, the separation between the top of each stage and the reference plane when the load is removed is different from the original separation. Let $\bar{h}_{i}$ denote the separation of the top of the $i$ th stage and the reference plane at the end of the unloading process. When the rigid plane is withdrawn to the $n$th stage that is above the critical stage, the total contact load can be expressed as

$$
P_{u}=\sum_{i=n+1}^{\infty} E s_{i}\left(\frac{u-u_{\max }}{H^{*}-h_{i}}+\varepsilon_{y}\right), u=\bar{h}_{n}<h_{n c} .
$$

Following the same procedure for analyzing the loading behavior, we obtain the total contact force during unloading as

$$
\frac{P_{u}}{P_{Y}}=\left\{\begin{array}{lr}
\frac{u}{\varepsilon_{y} H^{*}}\left(\frac{1}{f_{x}^{n+1}}-\frac{1}{f_{x}^{\bar{n}_{c}+1}}\right)-\frac{h_{0}\left(f_{x}-1\right)}{\varepsilon_{y} H^{*} f_{x}}\left(\frac{\beta^{n+1}-\beta^{\bar{n}_{c}+1}}{1-\beta}\right)+\frac{1}{\varepsilon_{y} f_{x}^{\bar{n}_{c}+1}}\left(\frac{u-u_{\max }}{H^{*}}+\varepsilon_{y}\right) & u=h_{n}, n \leqslant \bar{n}_{c} \\
\frac{1}{\varepsilon_{y} f_{x}^{n+1}}\left(\frac{u-u_{\max }}{H^{*}}+\varepsilon_{y}\right) & u=\bar{h}_{n}, \bar{n}_{c}<n
\end{array}\right.
$$


3.3 Asymptotic Analysis. In this section, we proceed to derive the asymptotic load-displacement expression. The difference between the heights of protrusions of the $i$ th and $(i+1)$ th stages diminishes as $i \rightarrow \infty$. Whence, the displacement can be treated as a continuous variable in the range $n \gg 1$. From the definition of Cantor set and the expression $u=h_{n}$, we obtain

$$
n=-\frac{\log \left(u / h_{0}\right)}{\log \left(f_{z}\right)}
$$

and

$$
\left(\frac{1}{f_{z}}\right)^{n}=\frac{u}{h_{0}} .
$$

Subsequently, it can be shown that for $n \gg 1$,

$$
\left(\frac{1}{f_{x}}\right)^{n}=\left(\frac{u}{h_{0}}\right)^{\alpha} \quad \text { and } \quad \beta^{n}=\left(\frac{u}{h_{0}}\right)^{1+\alpha},
$$

in which

$$
\alpha=\frac{\log \left(f_{x}\right)}{\log \left(f_{z}\right)} .
$$

Then we need to know the relationship between the critical stage $n_{c}$ and the displacement of the rigid half-space. When the rigid surface reaches the $n$th stage of asperities $\left(u=h_{n}\right)$, the asperities above the $n_{c}$ th stage deform plastically while those below the critical stage deform elastically. In other words, $\varepsilon_{n_{c}}<\varepsilon_{y}$ $\leqslant \varepsilon_{n_{c}+1}$. Using Eqs. (2) and (12), we obtain

$$
\left(\frac{1}{f_{z}}\right)^{n_{c}+1} \leqslant \frac{h_{n}-H^{*} \varepsilon_{y}}{h_{0}\left(1-\varepsilon_{y}\right)}=\frac{u-H^{*} \varepsilon_{y}}{h_{0}\left(1-\varepsilon_{y}\right)}<\left(\frac{1}{f_{z}}\right)^{n_{c}} .
$$

Let

$$
\chi=\frac{u-H^{*} \varepsilon_{y}}{h_{0}\left(1-\varepsilon_{y}\right)} .
$$

Equation (29) can be rewritten as

$$
n_{c}<-\frac{\log (\chi)}{\log \left(f_{z}\right)} \leqslant n_{c}+1
$$

As can be seen from Eq. (29), if $u / H^{*}<\varepsilon_{y}, n_{c}$ does not exist. This indicates that the deformation is purely elastic. On the other hand, if $u / H^{*} \gg \varepsilon_{y}, n_{c} \approx n+1$. This implies that the $(n+1)$ th stage yields when the rigid surface reaches the $n$th stage. Hence, the fractal rough surface almost has purely plastic deformation. For asymptotic analysis define

$$
n_{c}=-\frac{\log (\chi)}{\log \left(f_{z}\right)}
$$

It can be shown that

$$
\left(1 / f_{x}\right)^{n_{c}}=\chi^{\alpha} \quad \text { and } \quad \beta^{n} c=\chi^{1+\alpha}
$$

After substituting the above relations into Eqs. (19) and (21), we obtain the following load-displacement relations for purely elastic deformation and elastic-plastic deformation, respectively. For purely elastic deformation,

$$
\frac{P}{P_{Y}}=\left(\frac{h_{0}}{\varepsilon_{y} f_{x} H^{*}}\right)\left[1-\frac{\left(f_{x}-1\right) \beta}{1-\beta}\right]\left(\frac{u}{h_{0}}\right)^{1+\alpha} .
$$

For elastic-plastic deformation,

$$
\begin{aligned}
\frac{P}{P_{Y}}= & \left(\frac{h_{0}}{\varepsilon_{y} H^{*} f_{x}}\right) \times\left\{\left(\frac{u}{h_{0}}\right)\left[\left(\frac{u}{h_{0}}\right)^{\alpha}-\chi^{\alpha}\right]\right. \\
& \left.-\frac{\left(f_{x}-1\right) \beta}{1-\beta}\left[\left(\frac{u}{h_{0}}\right)^{1+\alpha}-\chi^{1+\alpha}\right]\right\}+\frac{\chi^{\alpha}}{f_{x}} .
\end{aligned}
$$

By a similar procedure, we proceed to analyze the asymptotical unloading behavior of the fractal surface. Let $u_{\max }$ denote the maximum displacement of the rigid plane, and $\bar{n}_{c}$ be the corresponding critical stage. From the definition of the critical stage, it can be shown that the distance between the top of the $\bar{n}_{c}$ th stage and the reference plane is

$$
\bar{h}_{\bar{n}_{c}}=h_{0} \chi_{M}
$$

where

$$
\chi_{M}=\frac{u_{\max }-\varepsilon_{y} H^{*}}{\left(1-\varepsilon_{y}\right) h_{0}} .
$$

The distance between the $\infty$-th stage and the reference plane due to the plastic deformation after the load is removed is

$$
\bar{h}_{\infty}=u_{\max }-\varepsilon_{y} H^{*} .
$$

During unloading, when the rigid plane is withdrawn to a position below the critical stage, the deformation of the fractal surface is partially plastic. On the other hand, when the rigid plane is withdrawn to a position above the critical stage, the fractal surface has purely plastic deformation. For these two cases, the loaddisplacement relationship during unloading can be, respectively, expressed as

$$
\frac{P}{P_{Y}}=\left\{\begin{array}{ll}
\frac{\left(u / h_{0}\right)}{\varepsilon_{y} f_{x}\left(H^{*} / h_{0}\right)}\left[\left(\frac{u}{h_{0}}\right)^{\alpha}-\chi_{M}^{\alpha}\right]-\frac{\left(f_{x}-1\right) \beta\left[\left(u / h_{0}\right)^{1+\alpha}-\chi_{M}^{1+\alpha}\right]}{\varepsilon_{y}\left(H^{*} / h_{0}\right) f_{x}(1-\beta)}+\left[\frac{\left(u / h_{0}\right)-\left(u_{\max } / h_{0}\right)}{\varepsilon_{y} H^{*} / h_{0}}+1\right] \frac{\chi_{M}^{\alpha}}{f_{x}} & h_{\bar{n}_{c}} \leqslant u<u_{\max } \\
\frac{1}{f_{x}\left(H^{*} / h_{0}\right)}\left(\frac{u}{\varepsilon_{y} h_{0}}-\frac{1-\varepsilon_{y}}{\varepsilon_{y}} \chi_{M}\right)^{\alpha+1} & \bar{h}_{\infty} \leqslant u<h_{\bar{n}_{c}}
\end{array} .\right.
$$

\section{Coefficient of Restitution}

Consider a rigid body with smooth flat contact surface approaching the stationary fractal surface with an initial velocity $v_{1}$. Following contact a short period of deformation takes place until the relative velocity between the two impacting bodies vanishes. At this instant, the fractal surface has maximum deformation, and the initial kinetic energy transforms into strain energy stored in the fractal surface if the energy contributed to the elastic wave propagation is negligible. During the remainder of contact a period of restitution occurs until the contact area reduces to zero. For a given incident velocity $v_{1}$, the maximum displacement of the rigid plane $u_{\max }$, at which the relative velocity between two impacting bodies vanishes, can be obtained by

$$
\frac{1}{2} m v_{1}^{2}=\int_{0}^{u_{\max }} P(u) d u,
$$

where $m$ is the mass of the rigid body and $P$ is the contact load during loading as described by Eq. (33) or (34), depending on whether plastic deformation is induced. For purely elastic contact, Eq. (33) should be employed. In this case, all the strain energy 
stored during loading will be recovered to kinetic energy. The rebound velocity of the rigid body at the end of the period of restitution is the same as the incident velocity. And the coefficient of restitution is unity. For a plastically deformed fractal surface, plastic deformation occurs during loading and only elastic deformation is involved during unloading, [1]. The strain energy released during unloading transforms into kinetic energy of the rigid body. Therefore the rebound velocity $v_{2}$ is obtained by

$$
\frac{1}{2} m v_{2}^{2}=\int_{h_{\infty}}^{u_{\max }} P_{u}(u) d u,
$$

where the unloading contact load $P_{u}$ is described by Eq. (38). The coefficient of restitution is obtained by

$$
e=\frac{v_{2}}{v_{1}}=\left(\frac{\int_{0}^{u_{\max }} P(u) d u}{\int_{\bar{h}_{\infty}}^{u_{\max }} P_{u}(u) d u}\right)^{1 / 2} .
$$

\section{Results and Discussion}

First, we verify the asymptotic load-displacement relationship. Figures $4(a)$ and $(b)$ show the comparison of the asymptotic (solid) and series (dashed) representations of the loaddisplacement relationship for various values of fractal parameters.

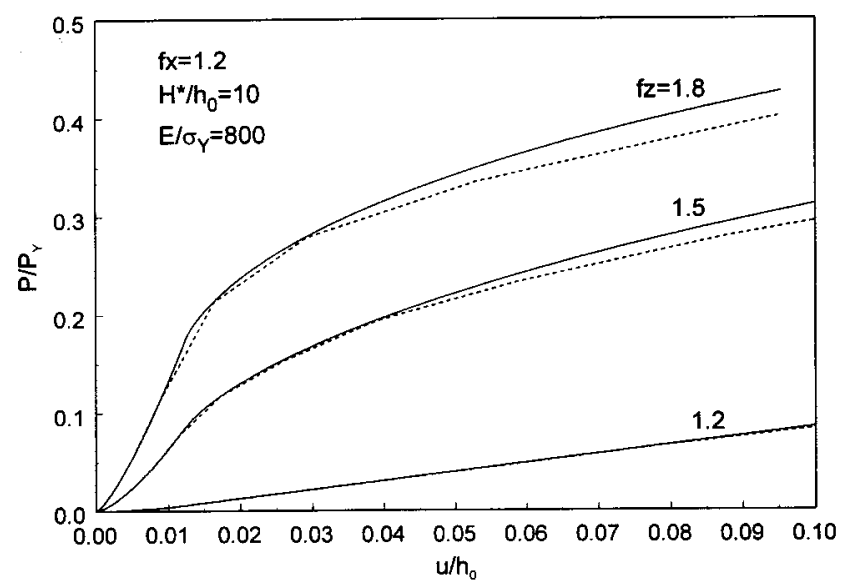

(a)

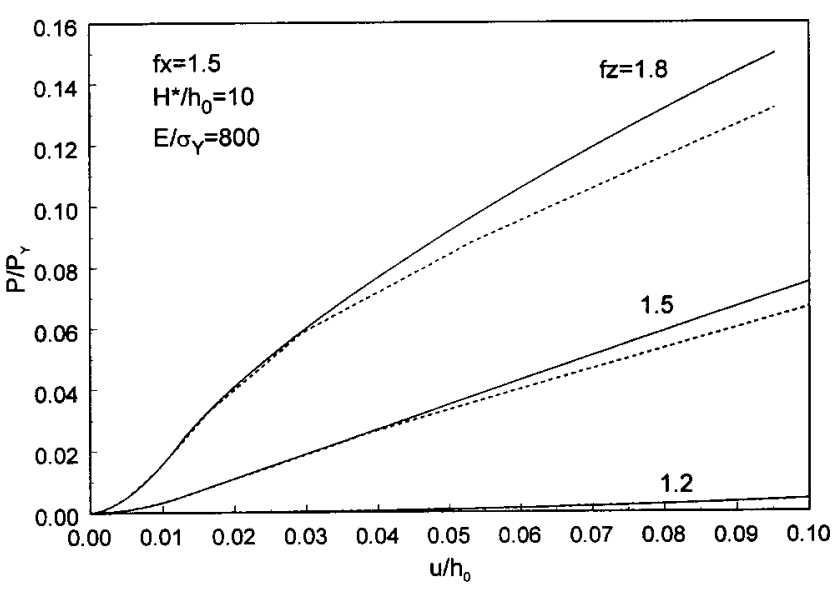

(b)

Fig. 4 Comparison of the asymptotic (solid) and series (dashed) results of the load-displacement curves for various values of fractal parameters, (a) $f_{x}=1.2$, (b) $f_{x}=1.5$

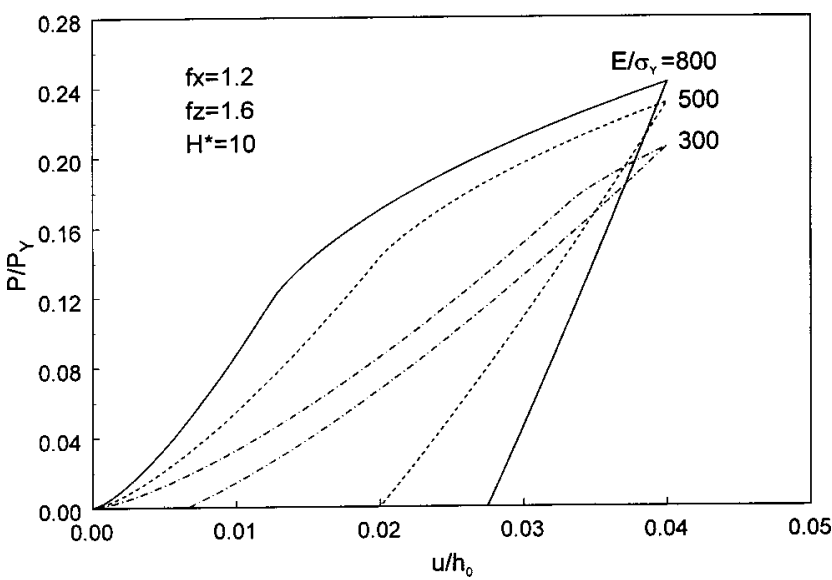

Fig. 5 Load versus displacement as a function of Young's modulus-to-yield stress ratio

As can be seen from the figures, the deviation between the asymptotic and series representations increases with the displacement, as expected. Besides, for a fixed value of $f_{x}$, the deviation between the asymptotic and series representations increases with $f_{z}$. It is observed that the deviation is less than $10 \%$ in the range $u / h_{0}<0.04$. In the following discussion, we confine the displacement in this range and use the asymptotic representations to study the effects of material properties and fractal parameters on the coefficient of restitution.

Figure 5 shows the load-unload curves at various Young's modulus-to-yield stress ratios. As can be seen from the figure, for a fixed value of displacement, the load increases with the Young's modulus, as expected. The unloading curves exhibit an initially linear response. This is due to the elastic recovery of the fractal surface. Bhattacharya and Nix [26] have shown that materials with a larger Young's modulus-to-yield stress ratio exhibit greater residual depth under a fixed maximum displacement, which is in agreement with the results shown in Fig. 5.

The significance of fractal parameters $f_{x}$ and $f_{z}$ on the loadunload curves is shown in Fig. 6. As can be seen from Fig. 6 that, when $f_{x}$ is held constant, the load required to produce the same displacement increases with $f_{z}$. This is due to the fact that a larger $f_{z}$ indicates a smoother surface. Equations (33) and (34) show that the parameter $\alpha=\log \left(f_{x}\right) / \log \left(f_{z}\right)$ has an import effect on the loading curve. Since $\alpha$ is positive, for purely elastic deformation, the load scales as the displacement to a power greater than one. Therefore, all the loading curves in Fig. 6 are initially convex. As the loading progresses, more and more asperities deform plastically and the last term in Eq. (34) becomes more significant. In this stage, the load scales as the displacement to a power greater than one for $f_{x}>f_{z}$, but less than one for $f_{x}<f_{z}$. It is observed in Fig. 6 that, as the loading progresses, the loading curve is entirely convex for $\alpha>1$, while changes from convex to concave for $\alpha$ $<1$.

Figure 7 shows the coefficient of restitution versus the dimensionless incident velocity for different values of the Young's modulus-to-yield stress ratio. The dimensionless incident velocity $V^{*}$ is defined as $V^{*}=\sqrt{m v^{2} /\left(2 l_{0} E h_{0}\right)}$. The coefficient of restitution is equal to unity when the incident velocity is lower than the critical velocity at which onset of plastic deformation occurs. The critical velocity decreases with the increasing Young's modulusto-yield stress ratio. Once the velocity exceeds the critical velocity, the coefficient of restitution is less than one because some energy is dissipated in plastic deformation. The area enclosed by the load-unload curve and the horizontal axis represents the energy lost in plastic deformation. As shown in Fig. 5, this area increases with the Young's modulus-to-yield stress ratio for a fixed value of maximum displacement. This is due to that most of 


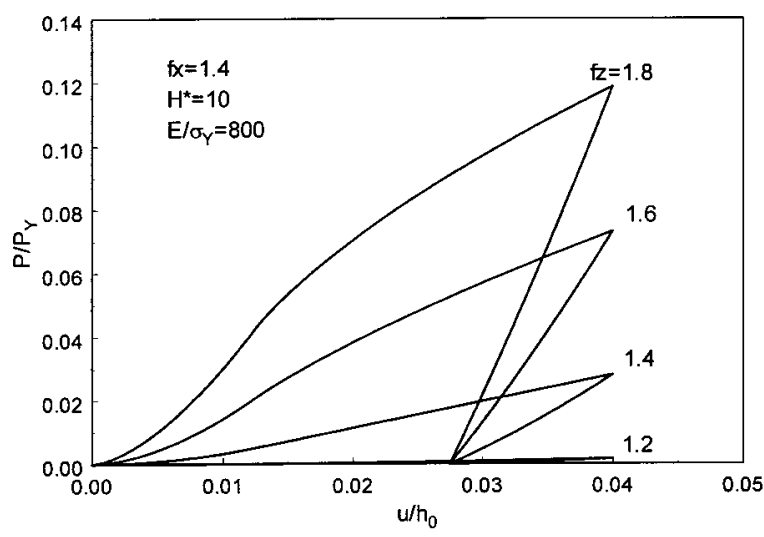

(a)

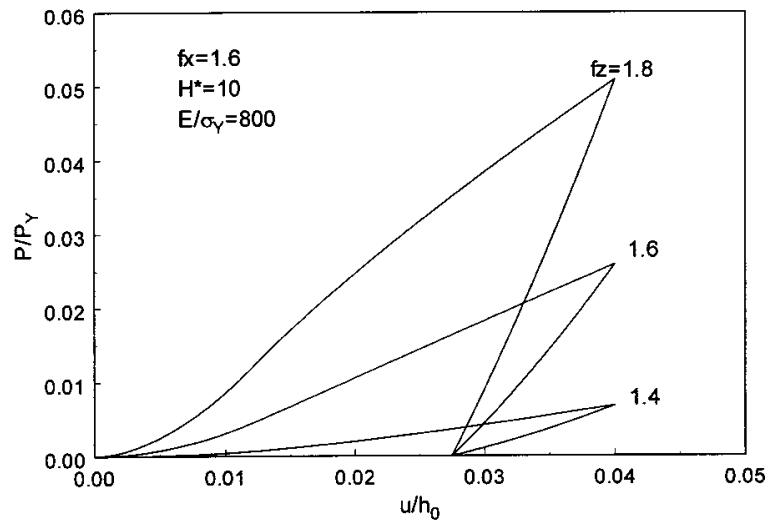

(b)

Fig. 6 Load-displacement curves for different values of fractal parameters, (a) $f_{x}=1.4$, (b) $f_{x}=1.8$

the asperities are deformed plastically when the Young's modulus to yield stress ratio is large. Figure 7 confirms that the coefficient of restitution decreases as the ratio of Young's modulus-to-yield stress is increased.

The effect of the fractal parameters on the coefficient of restitution is shown in Fig. 8. Figure $8(a)$ shows the coefficient of restitution versus the incident velocity for various values of $f_{z}$ while $f_{x}$ is held constant. The critical velocity increases with $f_{z}$. Once the incident velocity exceeds the critical velocity, the coef-

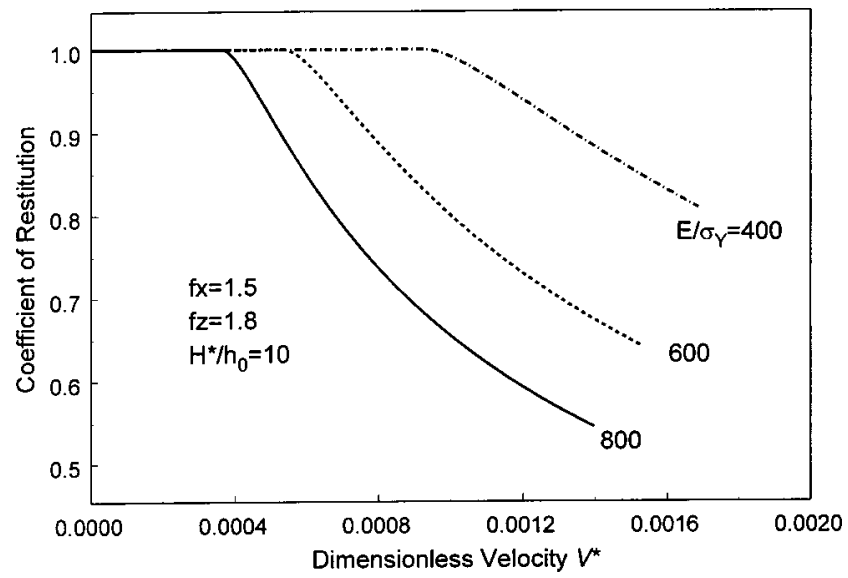

Fig. 7 Coefficient of restitution versus incident velocity as a function of Young's modulus-to-yield stress ratio

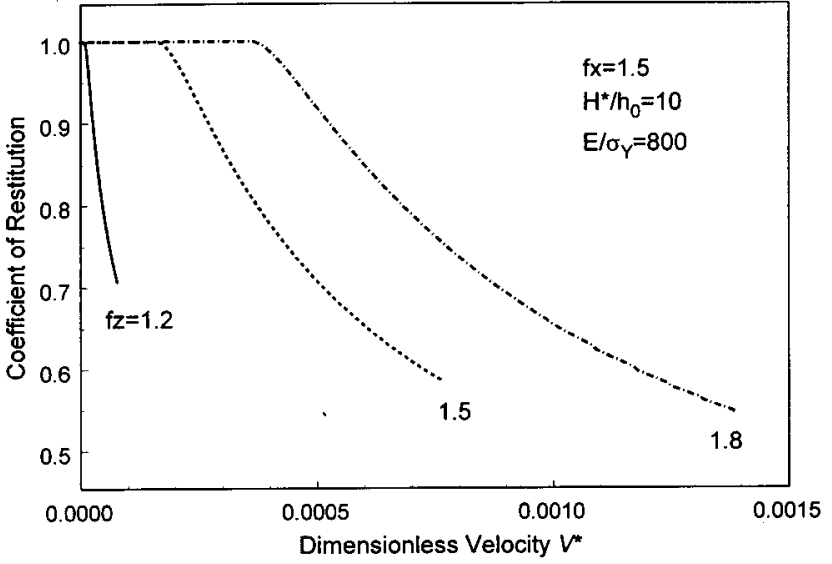

(a)

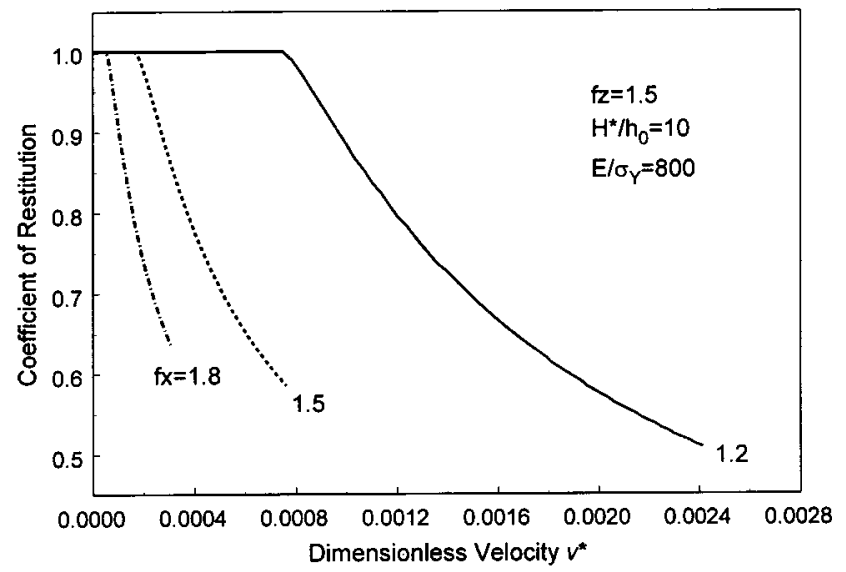

(b)

Fig. 8 Coefficient of restitution versus incident velocity for different values of fractal parameters, (a) $f_{x}=1.5$, (b) $f_{z}=1.5$

ficient of restitution decreases rapidly with the incident velocity. As incident velocity increases, the coefficient of restitution becomes less sensitive to velocity. A similar trend can be seen from Fig. $8(b)$ that the coefficient of restitution decreases with increasing $f_{x}$.

\section{Conclusion}

We studied the effect of surface roughness on the coefficient of restitution. We proposed an elastic-perfectly plastic contact model for a fractal surface in contact with a rigid smooth half-space. The fractal surface was constructed based on the Cantor set. Asymptotic analysis was performed to derive a continuous loaddisplacement relation. The result shows that the compliance relation depends strongly on the fractal parameters that characterize the surface topography. Based on the load-displacement relation, we investigated the significance of fractal parameters as well as material properties and incident velocity on the coefficient of restitution. It is found that materials with high Young's modulus-toyield stress ratio have smaller coefficient of restitution. Upon the point the incident velocity exceeds the critical velocity, the coefficient of restitution drops fast with the incident velocity. The sensitivity of the coefficient of restitution to the incident velocity decreases as the incident velocity increases. The coefficient of restitution decreases with increasing fractal dimension. 


\section{References}

[1] Tabor, D., 1951, The Hardness of Metals, Oxford University Press, London.

[2] Goldsmith, W., 1960, Impact, Arnold, London.

[3] Johnson, K. L., 1985, Contact Mechanics, Cambridge University Press, Cambridge, UK.

[4] Ling, F. F., 1958, “On Asperity Distributions of Metallic Surface,” J. Appl. Phys., 29, pp. 1168-1174.

[5] Nayak, P. R., 1971, "Random Process Model of Rough Surfaces," ASME J. Tribol., 93, pp. 398-407.

[6] Greenwood, J. A., and Williamson, J. B. P., 1966, "The Contact of Nominally Flat Surfaces," Proc. R. Soc. London, Ser. A, A295, pp. 300-319.

[7] Whitehouse, D. J., and Archard, J. F., 1970, "The Properties of Random Surfaces of Significance in Their Contact," Proc. R. Soc. London, Ser. A, A316, pp. $97-121$.

[8] Bush, A. W., Gibson, R. D., and Thomas, T. R., 1975, "The Elastic Contact of a Rough Surface," Wear, 35, pp. 87-111.

[9] Yamada, K., Takeda, N., Kagami, J., and Naoi, T., 1978, "Mechanisms of Elastic Contact and Friction Between Rough Surfaces," Wear, 48, pp. 15-34.

[10] Bhushan, B., 1984, "Analysis of the Real Area of Contact Between a Polymeric Magnetic Medium and a Rigid Surface," ASME J. Tribol., 106, pp 26-34.

[11] McCool, J. I., 1986, "Comparison of Models for the Contact of Rough Surfaces," Wear, 107, pp. 37-60.

[12] Chang, W. R., Etsion, I., and Body, D. B., 1987, "An Elastic-Plastic Model for the Contact of Rough Surfaces,” ASME J. Tribol., 109, pp. 257-263.

[13] Chang, W.-R., and Ling, F. F., 1992, "Normal Impact Model of Rough Surfaces," ASME J. Tribol., 114, pp. 439-447.

[14] Sayles, R. S., and Thomas, T. R., 1979, "Measurements of the Statistical
Microgeometry of Engineering Surfaces," ASME J. Lubr. Technol., 101, pp. 409-418.

[15] Greenwood, J. A., 1984, “A Unified Theory of Surface Roughness,” Proc. R. Soc. London, Ser. A, A393, pp. 133-157.

[16] Ling, F. F., 1989, "The Possible Role of Fractal Geometry in Tribology," Tribol. Trans., 32, pp. 497-505.

[17] Majumdar, A., and Bhushan, B., 1990, "Role of Fractal Geometry in Roughness Characterization and Contact Mechanics of Surfaces," ASME J. Tribol., 112, pp. 205-216.

[18] Mandelbrot, B. B., 1983, The Fractal Geometry of Nature, Freeman, New York.

[19] Kaplan, T., and Gray, L. J., 1985, "Effect of Disorder on a Fractal Model for the AC Response of a Rough Interface," Phys. Rev. B, 32, pp. 7360-7366.

[20] Church, E. L., 1988, "Fractal Surface Finish," Appl. Opt., 27, pp. 1518-1526.

[21] Majumdar, A., and Bhushan, B., 1991, "Fractal Model of Elastic-Plastic Contact Between Rough Surfaces,” ASME J. Tribol., 113, pp. 1-11.

[22] Wang, S., and Komvopoulos, K., 1994, "A Fractal Theory of the Interfacial Temperature Distribution in the Slow Sliding Regime: Part I-Elastic Contact and Heat Transfer Analysis," ASME J. Tribol., 116, pp. 812-823.

[23] Borodich, F. M., and Mosolov, A. B., 1992, "Fractal Roughness in Contact Problems," J. Appl. Math. Mech., 56, pp. 681-690.

[24] Warren, T. L., Majumdar, A., and Krajcinovic, D., 1996, “A Fractal Model for the Rigid-Perfectly Plastic Contact of Rough Surfaces,” ASME J. Appl. Mech., 63, pp. 47-54.

[25] Cook, R. D., and Young, W. C., 1985, Advanced Mechanics of Materials, Macmillan, New York.

[26] Bhattacharya, A. K., and Nix, W. D., 1988, "Finite Element Simulation of Indentation Experiments," Int. J. Solids Struct., 24, pp. 881-891. 